\title{
E-PBL : Ferramenta de Apoio ao aprendizado e uso da metodologia de Aprendizado Baseado em Problemas
}

\author{
Letícia Moura de Almeida, Marcos Paulo Sarges Rolim, Rodrigo Oliveira da Silva, \\ Anderson Jorge Serra da Costa \\ CCNT - Universidade do Estado do Pará (UEPA) - Castanhal - PA - Brasil \\ leticiamalmeida27@gmail.com, marcosbook98@gmail.com, \\ rodrigo.silval12@hotmail.com, andersonjsc@uepa.br
}

\begin{abstract}
This article focuses on the specification and development of a tool to support the application and learning of the active methodology of Problem Based Learning, and its main objective is to be a facilitator of learning and to encourage the use of this methodology. In the course of this work, a flowchart containing the main activities of the methodology. After identifying the characteristics of the methodology, software engineering techniques were applied to the system modeling and system development resulting from this work.
\end{abstract}

Resumo. Este artigo tem como foco a especificação e desenvolvimento de uma ferramenta de apoio à aplicação e aprendizado da metodologia ativa de Aprendizado Baseado em Problemas, e tem por objetivo principal ser um meio facilitador do aprendizado, e incentivar o uso dessa metodologia. No decorrer deste trabalho foi desenvolvido um fluxograma contendo as principais atividades da metodologia. Após a identificação das características da metodologia foram aplicadas técnicas da Engenharia de Software para a modelagem do sistema e desenvolvimento do sistema resultante deste trabalho.

\section{Introdução}

A área de Tecnologia da Informação (TI) possui como uma das principais atividades o desenvolvimento de software. Neste sentido, os programadores são fundamentais à TI, e por isso, a formação desses profissionais é de extrema importância. De acordo com Guedes e Paterno (2018), os estudantes geralmente apresentam problemas em entender a lógica de programação e, por consequência, ensinar e aprender programação torna-se um trabalho complexo.

Existem fatores que dificultam o aprendizado de programação de computadores, como o uso de metodologias tradicionais. Segundo Souza e Dourado (2015), nas metodologias tradicionais "o professor é o centro do processo de transmissão de saberes para alunos que apenas recebem e memorizam o conhecimento". Segundo Barros et al. (2017), em metodologias tradicionais, o ensino prático é pouco trabalhado, dificultando a fixação do conhecimento. No entanto, o docente deve articular as formas de ensinar, com vista, segundo 
VIII Congresso Brasileiro de Informática na Educação (CBIE 2019)

Anais do XXV Workshop de Informática na Escola (WIE 2019)

Souza e Dourado (2015), na construção de conteúdos pertinentes para a formação desse futuro profissional.

Farias et al. (2018) informam que diversas instituições de ensino superior (IES) adotam metodologias ativas como base curricular ou em disciplinas isoladas, sendo o Aprendizado Baseado em Problemas (do inglês Problem Based Learning - PBL) a proposta mais utilizada. A PBL foi desenvolvida por Howard Barrows em 1980, idealizada para a área da saúde, mas que tem crescido em outras áreas do ensino.

No entanto, há dificuldades em aplicar essa metodologia, já que o docente deve dominar o conteúdo e elaborar problemas do mundo real (BERBEL, 1998; SOUZA \& DOURADO, 2015; BARROS et al. 2017; RIBEIRO et al. 2018). Observou-se, também, que embora existam muitas publicações relacionadas ao PBL, segundo Farias et al. (2018) muitas apresentam ferramentas que não podem ser aplicadas em outros cenários. Com isso, definiuse a seguinte questão de pesquisa: como é possível facilitar o acesso dos docentes à metodologia PBL?

Considerando as dificuldades inerentes ao aprendizado de programação e as deficiências nas metodologias utilizadas, o principal objetivo deste trabalho é desenvolver uma ferramenta computacional destinada aos Smartphones. Que tem como proposta a redução do atrito inicial aluno - conteúdo, auxiliando no aprendizado e incentivando a utilização da metodologia PBL.

\section{Procedimentos Metodológicos}

Este trabalho consiste em uma pesquisa exploratória, com o objetivo de proporcionar maior familiaridade com o problema, a fim de torná-lo mais explícito (Gil, 2007). Esta pesquisa desenvolveu uma ferramenta no formato de um aplicativo para Smartphone, que permite a criação de planos de aula, seguindo uma estrutura que pode ser utilizada em diversos temas.

\subsection{Levantamento Bibliográfico}

Realizou-se um levantamento bibliográfico tradicional da literatura, com os termos "Problem Based Learning Applications", "Aprendizado Baseado em Problemas" e "Metodologias Ativas" nos repositórios Google Acadêmico, Anais do CSBC e Science Direct, visando encontrar estudos de aplicação do PBL e diferentes ferramentas. 
VIII Congresso Brasileiro de Informática na Educação (CBIE 2019)

Anais do XXV Workshop de Informática na Escola (WIE 2019)

\subsection{Desenvolvimento do sistema}

Para realizar a modelagem do sistema foram utilizados os diagramas de Casos de Uso, Classes e Atividades da linguagem UML, versão 2.0, na ferramenta Astah Community, versão 7.2.0, por ser gratuita e possuir as funcionalidades necessárias para a construção desses diagramas.

O sistema proposto por esse trabalho foi modelado com base (Woods, 2003) acerca do método PBL, demonstrada nas Figuras 1.
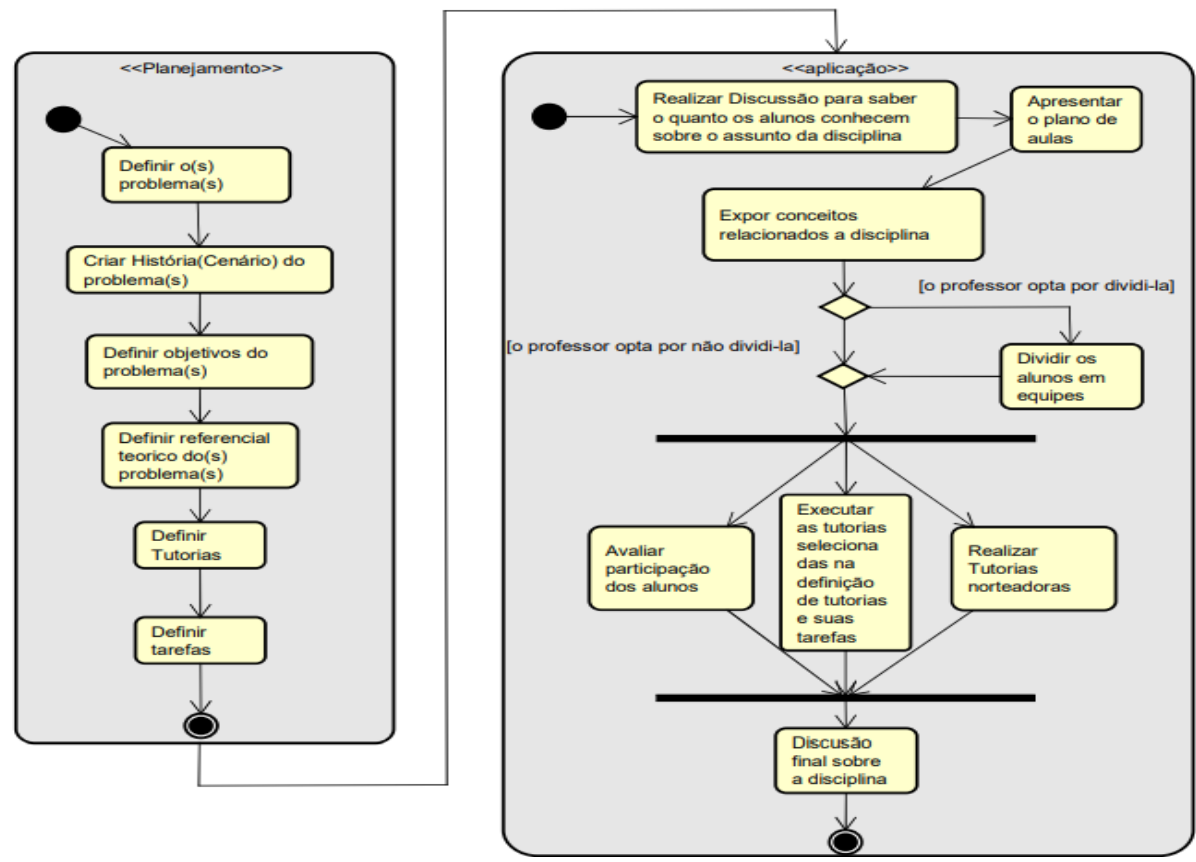

Figura 1. Diagrama de atividades do Fluxo - Fonte: Autoral

O sistema permite criação de planos de aula, baseados na metodologia PBL, além de um manual simplificado com respostas a possíveis dúvidas sobre a utilização da metodologia e sobre a própria ferramenta. Outras funcionalidades importantes são: a) Informações que detalham o plano de aula durante a sua criação como o problema, referências teóricas deste problema e os objetivos a serem alcançados durante a aplicação do plano de aula. b) Orientações para o preenchimento dos campos e sua relevância para a metodologia. c) Um exemplo preenchido da tela de criação do plano de aula.

A figura 2 apresenta a tela de acompanhamento do plano de aula, em a) Detalhes do direcionamento do plano de aula, a turma para a qual esse plano está direcionado, além de uma descrição sobre o problema, objetivos gerais e referências. Em b) Tutorias cadastradas no plano de aula e suas tarefas que podem ser editadas, marcadas como feitas ou desmarcadas. Dessa forma, o tutor poderá sempre verificar o que já foi trabalhado e o que ainda precisa ser 
VIII Congresso Brasileiro de Informática na Educação (CBIE 2019)

Anais do XXV Workshop de Informática na Escola (WIE 2019)

executado para finalizar o plano de aula. E em c) Orientações do que pode ser feito na área das tutorias.

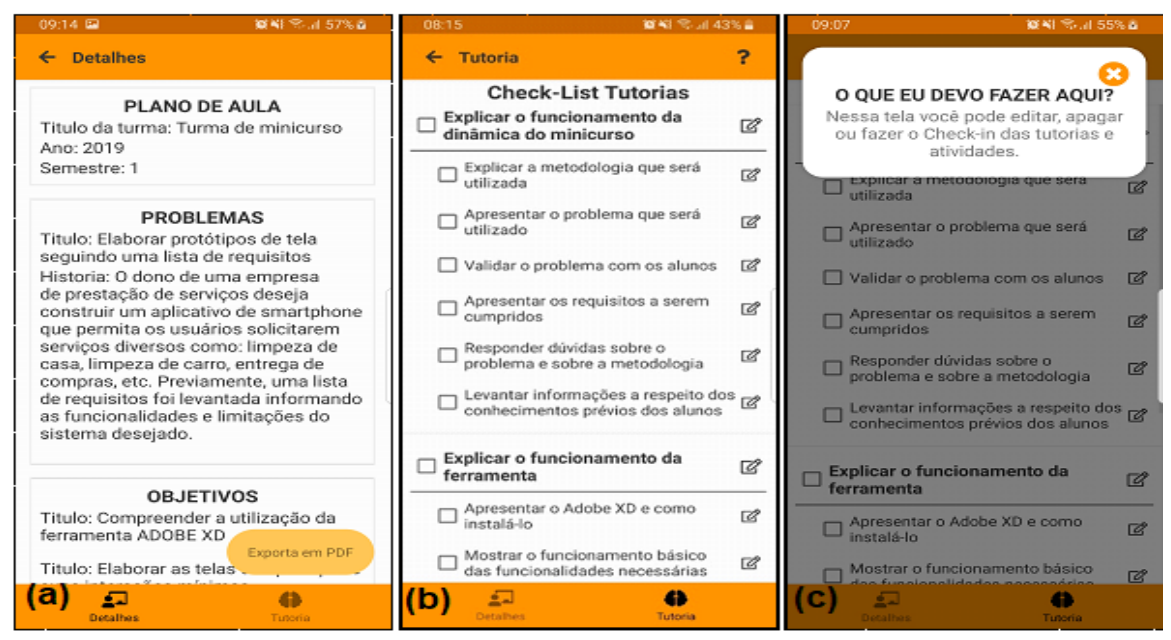

Figura 2. Tela de acompanhamento - Fonte: Autoral

O sistema desenvolvido não cobre todos os checklists sugeridos em (Woods, 2003), por conta de que alguns deles não foram considerados relevantes para iniciantes, pois exige aplicação anterior. Portanto, considera-se o trabalho relevante para a compreensão de como se utiliza a metodologia PBL.

\section{Considerações Finais}

A partir da realização desta pesquisa, pode-se concluir que houve sucesso durante o processo de construção da ferramenta. Vale ressaltar, que durante o período de criação do aplicativo houve dificuldades, por se tratar de uma pesquisa experimental no meio acadêmico. O que resultou na conclusão parcial dos objetivos traçados, fazendo com que as etapas não contempladas neste trabalho, se tornem sugestões para trabalhos futuros.

Aponta-se então, que a ferramenta ainda apresenta certas limitações, como a não realização de todos os checklists propostos nos estudos de (Woods, 2003) para uma melhor abordagem do PBL. Outro ponto, importante foi a dificuldade em adaptar o PBL, por se tratar de uma metodologia complexa e variada.

Neste sentido, a ferramenta desenvolvida apresenta um modelo reduzido, mas que pode servir de base e/ou ser reaproveitada, assim como, pode estimular novos projetos conceituais e práticos, com propostas similares, de aperfeiçoamento e aprofundamento. Que realizem aplicação e testes de qualidade, a fim de encontrar oportunidades de melhorias. Diante disso, espera-se que este trabalho auxilie o público alvo e seus demais usuários. 
VIII Congresso Brasileiro de Informática na Educação (CBIE 2019)

Anais do XXV Workshop de Informática na Escola (WIE 2019)

\section{Referências}

BARROS, F. L. F.; BITTENCOURT, R. A.; GONÇALVES, M. D.; SOUZA, S. M. (2017). Percepções Sobre Metodologias Ativas de Aprendizagem de Programação no Ensino Profissionalizante. Disponível em $\quad<\mathrm{http}$ :/www.brie.org/pub/index.php/wcbie/article/view/7502 >. Acesso em: 30 jun. 2019.

BERBEL, N. A. N. (1998). A problematização e a aprendizagem baseada em problemas: diferentes termos ou diferentes caminhos? Disponível em: $<$ http://www.scielo.br/pdf/icse/v2n2/08.pdf $>$

FARIAS, G.; FARIAS, N. M. M. Brito.; SPANHOL, F. J.; SOUZA, M. V. (2018). Moodle como Ferramenta de Suporte a PBL em Rede: Uma Revisão Sistemática. Disponível em: $<$ https://www.researchgate.net/publication/326644594_Moodle_como_Ferramenta_de_S uporte_a_PBL_em_Rede_Uma_Revisao_Sistematica>

GIL, A. C. Como elaborar projetos de pesquisa. 4. Ed. - reimpr. - São Paulo: Atlas, 2007. Disponivel em https://professores.faccat.br/moodle/pluginfile.php/13410/mod_resource/content/1/como_ elaborar_projeto_de_pesquisa_-_antonio_carlos_gil.pdf $>$.

GUEDES, L. R.; PATERNO, A. S. (2018). BrC: Proposta de uma Biblioteca em Português para Ensino de Programação em Linguagem C. Workshop sobre Educação em Computação (WEI_CSBC), v. 26, n. 1/2018. ISSN 2595-6175. Disponível em: $<$ http://portaldeconteudo.sbc.org.br/index.php/wei/article/view/3523>. Acesso em: 2018.

RIBEIRO, A. L.; BITTENCOURT, Roberto A.; SANTANA, Bianca L. (2018). Análise da Motivação em um Estudo Integrado de Programação Baseado em PBL. Disponível em: $<$ https://www.researchgate.net/publication/325054705_Analise_da_Motivacao_em_um_ Estudo_Integrado_de_Programacao_Baseado_em_PBL>

SOUZA, S. C.; DOURADO, L. (2015). Aprendizagem Baseada em Problemas (ABP): Um método de Aprendizagem inovador para o Ensino Educativo. Disponível em: $<$ http://www2.ifrn.edu.br/ojs/index.php/HOLOS/article/view/2880>

WOODS, Donald R. Preparing for PBL. Hamilton, ON: McMaster University, 2003. 